\title{
Impact of Sample Preservation and Manipulation on Insect Gut Microbiome Profiling. A Test Case With Fruit Flies (Diptera, Tephritidae)
}

\author{
Maarten De Cock ${ }^{1,2 *}$, Massimiliano Virgilio ${ }^{,}$, Peter Vandamme², Antonios Augustinos ${ }^{3}$, \\ Kostas Bourtzis ${ }^{4}$, Anne Willems ${ }^{2}$ and Marc De Meyer ${ }^{1}$
}

1 Department of Biology and Joint Experimental Molecular Unit, Royal Museum for Central Africa, Tervuren, Belgium, ${ }^{2}$ Laboratory of Microbiology, Faculty of Sciences, Ghent University, Ghent, Belgium, ${ }^{3}$ Department of Plant Protection, Institute of Industrial and Forage Crops, Hellenic Agricultural Organization - Demeter, Patras, Greece, ${ }^{4}$ Insect Pest Control Laboratory, Joint FAO/IAEA Programme of Nuclear Techniques in Food and Agriculture, Vienna, Austria

\section{OPEN ACCESS}

Edited by:

Brian Weiss,

Yale University, United States

Reviewed by:

Polychronis Rempoulakis, NSW Government, Australia

Panagiota Stathopoulou, University of Patras, Greece

*Correspondence:

Maarten De Cock

maarten_de_cock@hotmail.com

Specialty section:

This article was submitted to

Systems Microbiology,

a section of the journal

Frontiers in Microbiology

Received: 18 March 2019

Accepted: 22 November 2019

Published: 13 December 2019

Citation:

De Cock M, Virgilio $M$, Vandamme $P$, Augustinos $A$,

Bourtzis $K$, Willems $A$ and De Meyer M (2019) Impact of Sample Preservation and Manipulation on Insect Gut Microbiome Profiling. A Test Case With Fruit Flies (Diptera,

Tephritidae).

Front. Microbiol. 10:2833. doi: 10.3389/fmicb.2019.02833
High-throughput sequencing (HTS) techniques are of great value for the investigation of microbial communities, and have been extensively used to study the gut microbiome. While most studies focus on the human gut, many others have investigated insects. However, because of the rapid spread of HTS techniques, a lot of variation exists in the protocols for sample preparation. In the present study, we investigated the impact of two widely adopted sample-processing procedures preceding library preparation, i.e., preservation of insect tissue in $70 \%$ ethanol $(\mathrm{EtOH})$ and sample dissection. We used the fruit fly Ceratitis capitata (Diptera: Tephritidae) as a model organism and set up two experiments, one comparing the effects of sample manipulation and preservation across life stages and the other across fruit samples from different sources. The results of this study showed no major effects of dissection on the outcome of HTS. However, $\mathrm{EtOH}$ preservation did have effects on the recovered gut microbiome, the main effect being a significant reduction of the dominant genus, Providencia, in EtOH-preserved samples. Less abundant bacterial groups were also affected resulting in altered microbial profiles obtained from samples preserved in $70 \% \mathrm{EtOH}$. These results have important implications for the planning of future studies and when comparing studies that used different sample preparation protocols.

Keywords: amplicon sequencing, gut microbiome, methodology, Tephritidae, Ceratitis capitata

\section{INTRODUCTION}

Microbial communities are an integral part of the functioning and survival of all ecosystems and all living organisms (Holguin et al., 2001; Rosenberg et al., 2007; Zilber-Rosenberg and Rosenberg, 2008). In recent decades, the emergence of high-throughput sequencing (HTS) techniques has revolutionized the study of these communities (Metzker, 2010; Morey et al., 2013). These methods allow large amounts of information about microbiological communities to be collected in a relatively short time, without the need of specialized microbiological techniques. However, the fast emergence of this technique led to a large diversity of protocols applied. 
Using HTS techniques, increasing amounts of information have been collected describing the gut microbiome and its potential benefits to the host fitness. While a lot of this research has been focused on the human gut microbiome (NIH HMP Working Group et al., 2009; Kinross et al., 2011), several studies have addressed other organisms, including insects (Dillon and Dillon, 2004; Alma, 2008; Engel and Moran, 2013; Raymann and Moran, 2018). These studies have linked microbiological activity to improved digestion of indigestible components, including toxins, increased reproductive output, and many other factors benefiting the host. However, recent studies have indicated potential large effects of different sample preparation protocols in the study of microbiological communities (Hale et al., 2015; Hammer et al., 2015; Song et al., 2016). In fecal samples, one of the most crucial aspect emerging from these studies is preservation, as multiple studies have reported large effects of different preservation protocols on the microbiological community (Vlčková et al., 2012; Hale et al., 2015; Song et al., 2016). Therefore, the use of a strictly standardized protocol for sample processing is of utmost importance.

Tephritid fruit flies (Diptera: Tephritidae) are well-known pests in agricultural- and horticultural crops. Worldwide there are more than 4600 species of fruit flies (White and ElsonHarris, 1992; Uchôa, 2014), of which a large part utilizes fruits for larval development, as suggested by their name. This causes enormous losses, both directly, by damaging the fruit tissue, and indirectly, by accelerating the rotting process and infestation by other insects, fungi, and bacteria. Because of this, infestations by fruit flies can have huge economic impacts on the agricultural sector. The Mediterranean fruit fly (Medfly), Ceratitis capitata, is one of the most wide spread and notorious fruit fly species (De Meyer et al., 2008; Dominiak and Daniels, 2012). While native to sub-Saharan Africa, it has spread throughout the Mediterranean region, Latin-America, and Western Australia, with occasional records from North-America (De Meyer et al., 2008). One of the main reasons behind this widespread occurrence is its highly polyphagous nature, enabling it to exploit a wide variety of plant species. The host species can thus vary depending on the available plant species in a particular region or time. Currently, C. capitata infestations have been found in more than 260 plant species. Many of these host species are agricultural crops, and therefore the control of these species is of uttermost importance. The gut microbiome of tephritid fruit flies has increasingly been studied in the recent years, revealing the presence and role of many microorganisms. Examples include bacteria helping to overcome pesticides (Cheng et al., 2017) and host defenses (Ben-yosef et al., 2015) or generally increasing longevity of fruit flies (Behar et al., 2008b). Nevertheless, the overall knowledge of the fruit fly microbiome is still very fragmented and lacking in many areas.

In the study of the insect gut microbiome variation in the preparation protocol has only been studied to a limited extent. Although, as for fecal samples, we can suspect effects of the choice of preparation protocol in this kind of samples, empirical evidence for this is still lacking. In the present study, we set out to analyze two aspects of sample processing for HTS study of the gut microbiome of insects, using C. capitata (Diptera: Tephritidae) as a model organism. The first aspect of the sample processing protocol that was analyzed is the effect of dissection of the gut from the insect body. The removal of the gut (or other insect organs) is a well-established part of the protocol of many gut microbiome studies (Husseneder and Grace, 2005; Ami et al., 2010; Gavriel et al., 2011; Colman et al., 2012; Augustinos et al., 2015; Clarke, 2016; Zhang et al., 2016). However, since dissecting the gut from bodies of tiny insects, such as fruit flies, is challenging and time consuming, this step is often omitted from the sample preparation protocol (Wong et al., 2011; CejaNavarro et al., 2015; Garofalo et al., 2017). Although dissection might have a large impact on the gut microbiome assemblage recovered, to our knowledge, no studies have previously assessed this effect. Our hypothesis is that dissection in fruit flies, and in particular larvae, has no significant effect on the gut microbiome profiles recovered through HTS and can therefore be omitted from the protocol. The second aspect that was studied is the effect of storing individual insects in $70 \%$ ethanol (EtOH). As $\mathrm{EtOH}$ is a product that is easily acquired and transported, it is ideal for the collection and storage of fruit flies, even in countries where fast cooling is less evident. Additionally, preserving insects in $70 \% \mathrm{EtOH}$ has the benefit of keeping insects flexible enough to make manipulations, such as removal of the gut, feasible, while this is not actually possible with specimens preserved in $100 \% \mathrm{EtOH}$, which become more fragile and tend to break during dissection. In fecal samples, 70\% EtOH has been reported to have some effect on the microbiome recovered and its use is advised against (Vlčková et al., 2012; Hale et al., 2015; Song et al., 2016). Therefore, identifying and quantifying how EtOH preservation affects the gut microbiome in insect bodies should provide valuable information. Our hypothesis is that preserving insect samples in $70 \% \mathrm{EtOH}$ for long periods will have no major effects on the gut microbiota and major patterns in microbiome composition will still emerge even after preservation.

Like many insects, tephritid fruit flies go through a significant metamorphosis during their development to adults. It has been shown in previous studies that this metamorphosis in insects, including tephritid fruit flies, can have major effects on the gut microbiome (Morales-Jiménez et al., 2012; Aharon et al., 2013; Andongma et al., 2015). Additionally, recent studies have shown that there are major differences in the gut microbiome between different populations within the same fruit fly species (Wang et al., 2011, 2014). In the present study, we aim at verifying if widely adopted insect preservation and manipulation procedures might significantly bias the HTS profiling of their gut microbial communities. This will provide important baseline information to interpret and compare data from different studies.

\section{MATERIALS AND METHODS}

\section{Experimental Setup}

In a first experiment, we investigated the effects of gut dissection and sample preservation across different life stages of C. capitata. Reared specimens were provided by the Insect Pest Control Laboratory (IPCL) of the Joint FAO/IAEA 
Division of Nuclear Techniques in Food and Agriculture, International Atomic Energy Agency (IAEA). We sampled thirdinstar larvae, tenerals (i.e., newly emerged adults) and mature adults from a long-established laboratory colony population ( $>30$ years; $>400$ generations) of Greek origin. More than 60 individuals were collected for each life stage, 30 of which were processed immediately (see below) and the remaining individuals were stored in $70 \% \mathrm{EtOH}$ at $-20^{\circ} \mathrm{C}$ for a period varying from 12 to 18 weeks.

In a second experiment we evaluated the effects of sample dissection across EtOH-preserved third-instar larvae from different sources. We sampled three different laboratory colonies of C. capitata, provided by the IPCL. This included the longestablished colony population ( $>30$ years; $>400$ generations) of Greek origin, an intermediate established colony population (eight generations) of Australian origin and a newly established colony population (one generation) of Argentinian origin. Larvae collected from a wild population from Italy were also included in the experimental setup. Before processing the wild population, the identity of each larva was confirmed via DNA barcoding (see Supplementary Table S1) as described in Virgilio et al. (2012). Each sample was composed of more than 30 larvae that were immediately stored in $70 \% \mathrm{EtOH}$ (see Supplementary Tables S2, S3 for details on the experimental design and sample collection).

\section{Laboratory Procedures}

Before sample processing, the body surface of all insect specimens was sterilized in $70 \% \mathrm{EtOH}$ for $30 \mathrm{~s}$ and then rinsed once in phosphate-buffered saline (PBS) water. Guts were dissected by removing, with sterilized tools, the whole gut from crop to anus. Undissected, full bodies were directly crushed with a sterilized pestle. In order to minimize biases due to interindividual variability, five dissected guts or crushed bodies were pooled per sample and DNA was extracted from each pool using the Qiagen DNAEasy extraction kit as per the manufacturer's protocol. Before genomic library preparation, DNA concentrations of samples were determined using a Qubit Fluorometer (Thermofisher). Samples with DNA concentration $<1 \mathrm{ng} / \mu \mathrm{l}$ were discarded and DNA extraction repeated on a novel set of specimens. The genomic library preparation targeted the V3-V4 region of the 16S ribosomal RNA gene [rRNA, insert size $465 \mathrm{bp}, 341 \mathrm{~F}$, and 806R primers (Takahashi et al., 2014)] and relied on the Nextera XT kit (including Illumina sequencing adapters, and dual-index barcodes) as per the manufacturer's protocol. DNA was amplified in two steps and, for most samples, the second amplification was repeated to increase DNA yield. A final quality check of fragment size distributions was performed using an Agilent 2100 Bioanalyzer system. A mock community including DNA of 18 bacterial strains from the BCCM/LMG Bacteria Collection ${ }^{1}$ and a blank were also included in the Illumina run as positive and negative controls, respectively. Libraries were sequenced on an Illumina MiqSeq platform [300 bp paired end (PE) sequencing, performed by Macrogen].

${ }^{1}$ http://bccm.belspo.be/

\section{Analysis of Data}

Read quality was preliminarily assessed in FastQC (Andrews, 2014) and data filtering implemented via the DADA2 pipeline (Callahan et al., 2016) in R. This pipeline is based on a selflearning algorithm that compiles a parametric error model fitting the raw data, which is then used to infer sequencing errors. After trimming, demultiplexing, and filtering, paired reads were assigned to operational Taxonomic Units (OTUs) according to the Bayesian classifier method implemented by DADA2 (Wang et al., 2007). Results for the mock community and blanks were used to determine quality of the analysis. The Silva reference database v132 (Pruesse et al., 2012) was used for taxonomic assignment of OTUs (percentage of identity $=97 \%$ similarity, $\mathrm{p}$-min-consensus $=0.51$ ) and the robustness of taxonomic assignment was double-checked using the RDP (Cole et al., 2014) and Greengenes databases (DeSantis et al., 2006; data not shown). The complete analytical pipeline is detailed in Supplementary Table S4. Before further analysis singletons and double tons were removed from the data and OTUs with a significant presence in the blanks were deleted. For comparison between samples data scaling, based on the median sample number of reads, was implemented (de Cárcer et al., 2011).

Downstream analyses were done in R, using the Phyloseq (McMurdie and Holmes, 2013) and Vegan (Oksanen et al., 2017) packages. Differences in univariate patterns of alpha diversity [as estimated by the Reverse Simpson index (Lande, 2016)], calculated from OTU data, were tested via analysis of variance (ANOVA), with life stage (larva, teneral, and adult), preservation (fresh vs. EtOH preserved), and dissection (dissected gut vs. full body) as fixed, orthogonal factors for the first experiment and dissection (dissected gut vs. full body) and sample origin (colony strain Greece, colony strain Australia, colony strain Argentina, wild population Italy) as fixed, and random orthogonal factors, respectively, for the second experiment. A posteriori pairwise comparisons of significant factors were implemented via Tukey's honestly significant difference (HSD) test (Abdi and Williams, 2010).

Multivariate differences in OTU abundance and composition were tested using permutational multivariate analysis of variance (PERMANOVA; Anderson, 2017) and permutational multivariate analysis of dispersion (PERMDISP; Anderson, 2006) on scaled data as implemented by the programs PERMANOVA and PERMDISP. For PERMANOVA we relied on the same threeway factorial setup described above for univariate analyses, while for PERMDISP, which only applies to two-ways experimental designs, we tested the effects of life stage and preservation. A posteriori pairwise comparisons of multivariate significant factors were then implemented using the permutational $t$-statistics of PERMANOVA and PERMDISP. Probability values of repeated a posteriori tests were corrected for Type I errors using the false discovery rate procedure (Benjamini and Hochberg, 1995) with experiment-wise probability $p=0.05$. Multivariate patterns were visually interpreted via scaled and centered principal coordinates analyses (PCoAs) based on Bray-Curtis distance (Bray and Curtis, 1957) as implemented by the R package ggplot2 (Wickham, 2009). 


\section{RESULTS}

\section{Overall Bacterial Diversity Associated With C. capitata}

The Illumina Miseq run yielded more than $13 \times 10^{6} \mathrm{PE}$ reads across the 54 samples considered. After assessing the quality of the reads in FastQC (Andrews, 2014), forward and reverse reads were trimmed to 230 and $200 \mathrm{bp}$, respectively. Based on read quality, a strict error rate $(\max N s=0, \max$ error rate $=1$, see Supplementary Table S4) was applied. After quality control, demultiplexing, pairing, and filtering, we obtained a total of $3.6 \times 10^{6}$ reads, corresponding to a total of 848 unique OTUs. Taxonomic assignment of OTUs yielded 155 genera from 13 phyla (Supplementary Table S6). The phylum Proteobacteria was by far the most dominant (91.64\% of reads) and comprised a few dominant genera: Acinetobacter (19.03\%), Pluralibacter (11.31\%), Morganella (7.40\%), Klebsiella (3.36\%), Serratia (2.06\%), and Enterobacter (1.44\%). The second most important phylum, Firmicutes (8.21\%), included different genera from the orders Bacillales [mainly represented by Bacillus (2.15\%), Staphylococcus (1.77\%), and Salinicoccus (1.19\%)], and Lactobacillales [mainly including Lactococcus $(1.08 \%)$ and Lactobacillus $(0.78 \%)]$. Genera from the phyla Bacteroidetes $(0.44 \%)$ and Actinobacteria $(0.22 \%)$ could also be identified. The remaining phyla represented $<0.01 \%$ of the total reads. A full overview of the OTU composition can be found in Supplementary Table S5. Comparison between samples used not the total read output as described above but a scaled dataset.

\section{Impact of Sample Preservation and Manipulation Across Life Stages}

On average, samples from the first experiment had 47.26 OTUs $(\mathrm{SD}=28.30)$ and a reverse Simpson index of $0.43(\mathrm{SD}=0.26)$. ANOVA on the reverse Simpson index obtained from the OTU data showed no effects of dissection or life stage on diversity (Table 1A and Supplementary Table S7a). However, a highly significant effect of preservation, with lower diversity in fresh compared to EtOH-preserved samples was found (Figure 1).

Permutational multivariate analysis of variance revealed a highly significant interaction of preservation and life stage (at $P<0.001)$ as well as a significant interaction of preservation and dissection (at $P=0.047$ ) (Table 1A and Supplementary Table S8a). The a posteriori comparisons showed that EtOH preservation always had a significant effect on the multivariate patterns of microbial assemblages. Interestingly, significant differences across life stages were detected across all EtOHpreserved specimens, while in the microbial assemblages of fresh specimens, significant variations were only observed when comparing tenerals to adults (Supplementary Table S8a). Effects of dissection were only detected in fresh samples $(P=0.040)$ (Supplementary Table S8a).

Permutational multivariate analysis of dispersion showed a significant interaction of life stage and preservation (Table 1A and Supplementary Table S9a). Pairwise a posteriori tests (Supplementary Table S9a) revealed that the multivariate patterns of dispersion of fresh samples were comparable
TABLE 1 | Summary table for ANOVA, PERMANOVA, and PERMDISP testing for differences in patterns of alpha diversity (as estimated by the Reverse Simpson index calculated from OTU data) across (A) dissection procedures, sample preservation methods, and life stages of $\mathbf{C}$. capitata and $\mathbf{( B )}$ dissection procedures on different populations of $C$. capitata.

\begin{tabular}{lccc}
\hline & ANOVA & PERMANOVA & PERMDISP \\
\hline (A) & & & \\
Life stage (li) & n.s. & $* * *$ & n.s. \\
Preservation (pr) & $* * *$ & $* * *$ & n.s. \\
Dissection (di) & n.s. & n.s. & \\
Li $\times$ pr & n.s. & $* * *$ & $* *$ \\
Li $\times$ di & n.s. & n.s. & \\
Pr $\times$ di & n.s. & n.s. & \\
Li $\times$ pr $\times$ di & n.s. & & \\
$(B)$ & & $* * *$ & n.s. \\
Origin (or) & n.s. & n.s. & n.s. \\
Dissection (di) & n.s. & n.s. & \\
Or $\times$ di & n.s. &
\end{tabular}

Full tables available in Supplementary Table S7. n.s., not significant; *, significant at $P<0.05$, ***, at $P<0.001$.

(with average within group dissimilarities ranging from 14.717 to 26.374) and not significantly different, while in EtOH-preserved samples the multivariate dispersion of tenerals, adults, and larvae significantly differed with average within group dissimilarities of, respectively, 58.335, 31.031, and 2.091.

Overall, the first two axes of the PCOA (Figure 2) explained $71.9 \%$ of variation (47.7 and $23.8 \%$ for PC1 and PC2, respectively). The visual inspection of graphs again suggested a lack of major differences between full body and dissected gut samples. EtOH preservation showed distinct differences where fresh individuals of all life stages combined, while EtOH-preserved samples form distinct groups corresponding to their life stage. Fresh samples of tenerals and adults were markedly less dispersed around their group centroid compared to their EtOH-preserved counterparts. Interestingly, fresh larvae have a remarkably higher dispersion compared to $\mathrm{EtOH}-$ preserved larvae.

The relative abundance of the most common taxa across treatments did not suggest any obvious effect of dissection in either fresh or EtOH-preserved samples (Supplementary Table S10). However, as indicated by PERMANOVA there were some effects of dissection within fresh samples. In taxon composition this was apparent as a lower dominance of Providencia in full body samples (gut: 93.84\%, SD: 7.00\%; full: $78.46 \%$, SD: $18.66 \%)$. Conversely, there was a slightly higher relative abundance of many other genera in full body samples. However, there were only two genera, Serratia and Klebsiella, where this difference exceeded $1 \%$ relative abundance. Conversely, EtOH preservation heavily affected the composition of the gut microbiome with not consistent effects from life stage to life stage (Figure 3). Overall, we could observe a general trend from dominance of Providencia in fresh samples (larvae: $92.57 \%$, SD: $12.81 \%$; tenerals: $80.66 \%$, SD: $19.27 \%$; adults: 85.22\%, SD: $15.04 \%$ ) to a strong decline of Providencia (larvae: 0.14\%, SD: $0.19 \%$; tenerals: $35.83 \%$, SD: $35.82 \%$; adults: $0.90 \%$, 


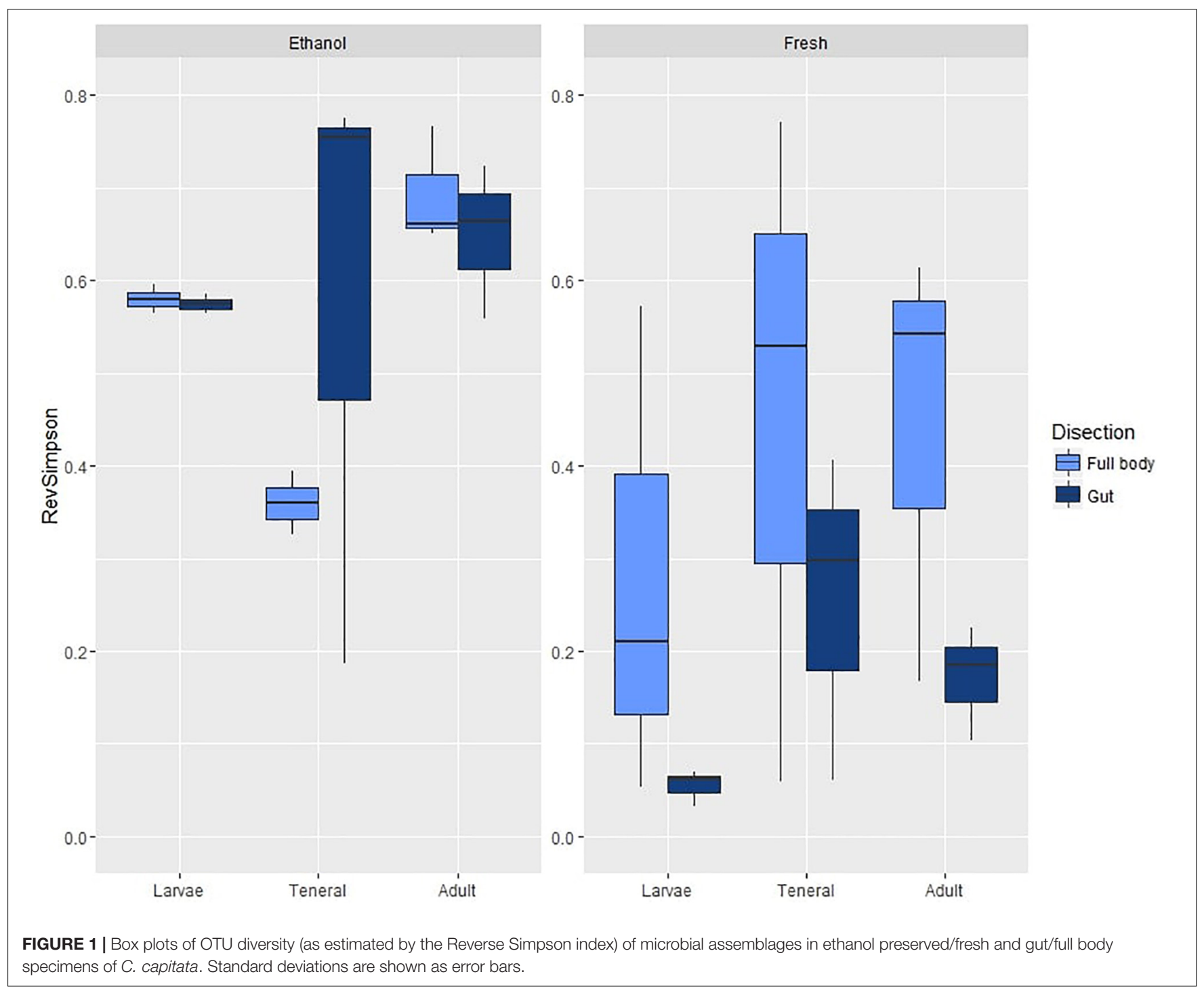

SD: $0.96 \%$ ) and dominance of one or more other genera in EtOH-preserved samples. In EtOH-preserved larvae, we observed Acinetobacter as the dominant genus (fresh: 0.05\%, SD: 0.05\%; EtOH: 98.63\%, SD: 1.11\%). In EtOH-preserved tenerals, Providencia (fresh: 80.66\%, 19.27\%; EtOH: 35.83\%, SD: $35.83 \%$ ), Salinicoccus (fresh: 1.35\%, SD: 1.1\%; EtOH: 32.60\%, SD: 27.23\%), and Staphylococcus (fresh: 1.92\%, SD: 1.74\%; EtOH: 23.29\%, SD: $35.59 \%)$ are the most abundant taxa. In EtOH-preserved adults, Pluralibacter (fresh: 0.15\%, SD: 0.2\%; EtOH: $61.41 \%$, SD: $11.45 \%$ ) was dominant, followed by Acinetobacter (fresh: $0.60 \%$, SD: $1.03 \%$; EtOH: 14.63\%, SD: $21.2 \%$ ), Serratia (fresh: $3.43 \%$, SD: 5.9\%; EtOH: 6.52\%, SD: 7.02\%), Klebsiella (fresh: 3.01\%, SD: 6.36\%; EtOH: 3.73\%, SD: 1.87\%), Cronobacter (fresh: 0.02\%, SD: $0.03 \%$; EtOH: 3.28\%, SD: 2.14\%), and Enterobacter (fresh: 0.01\%, SD: 0.01\%, EtOH: 2.96\%, SD: 2.18\%). Additionally, even if the qualitative composition of within group replicates was remarkably similar (particularly for the dominant groups, see Supplementary Table S10), quantitative differences and differences of low abundant genera could also be observed (see error bars of Figure 3). Details about the taxa relative abundance and variability are provided in Supplementary Table S10.

\section{Impact of Sample Manipulation Across Fruit Fly Samples From Different Sources}

For the second experiment, we observed an average diversity per sample of 44.42 OTUs $(S D=19.62)$ and an average reverse Simpson index of 0.57 (SD =0.20). ANOVA (Table $\mathbf{1 B}$ and Supplementary Table S7b) did not show significant differences in species diversity between dissection methods or across populations (Figure 4).

However, PERMANOVA (Table 1B and Supplementary Table S8b) did reveal significant variability across populations from different origin, with significant differences in all pairwise comparisons, while it did not detect differences between the multivariate patterns of dissected and non-dissected samples.

Similarly, PERMDISP (Table 1B and Supplementary Table S9b) showed highly significant differences across 


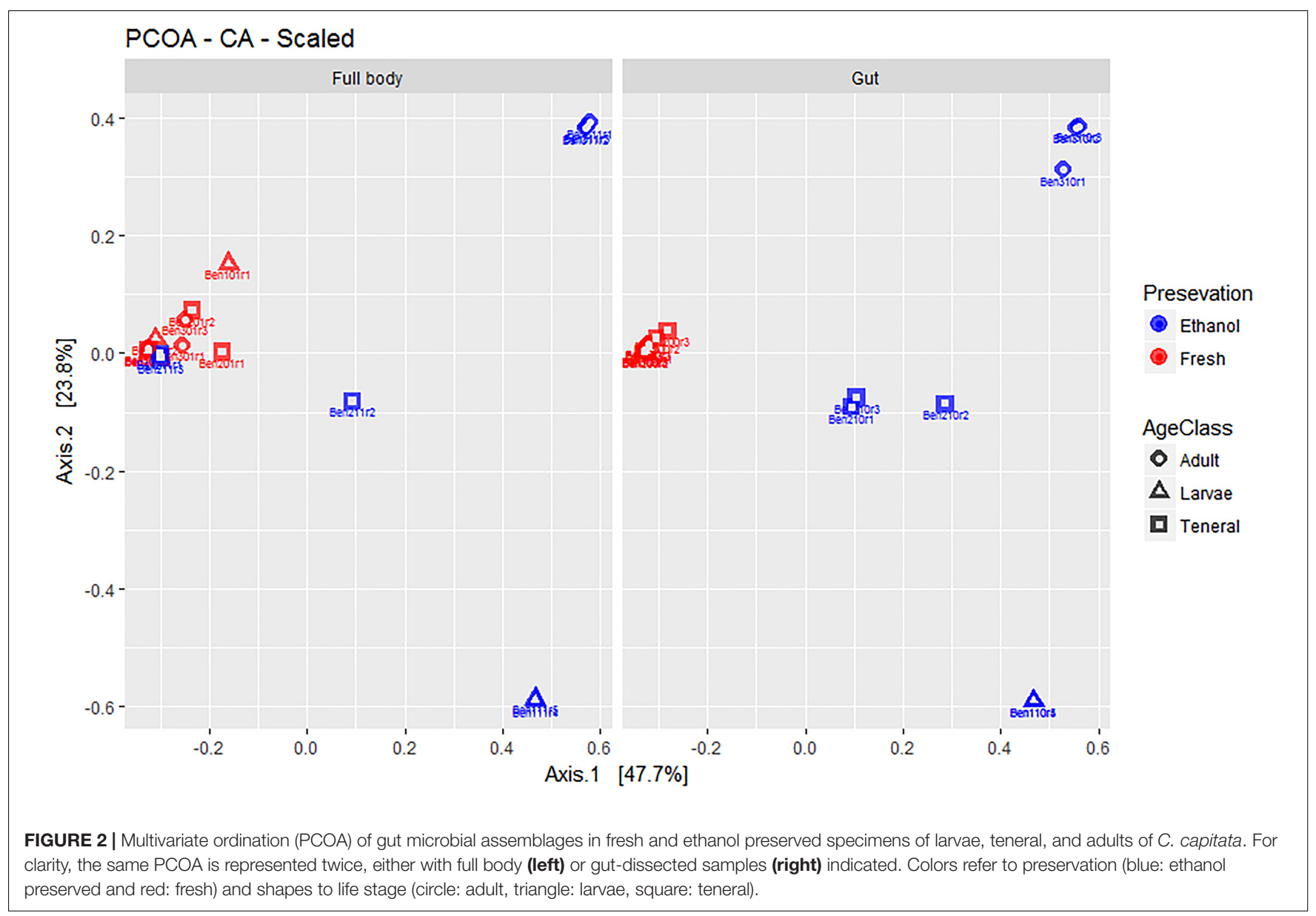

populations, with average within group dissimilarities ranging from 16.824 (Greece) to 76.975 (Argentina). Significant differences occurred in comparisons between all populations, except between the Italian and Argentinian population. PERMDISP also did not detect significant differences between dissected guts or full bodies.

Overall, the first two axes of the PCOA (Figure 5) explained $54.3 \%$ of variation (30.1 and $24.1 \%$ for PC1 and PC2, respectively). The visual analysis of Figure 5 further suggested the lack of relevant differences between the microbial assemblages obtained from full body and gut-dissected samples. Populations from Greece (with remarkably low dispersion around the corresponding group centroid) and Australia formed separate groups while the Argentinian and Italian populations grouped closer together.

Analyzing the taxon compositions across populations (Figure 6) further confirmed minor differences related to the dissection protocol. The microbiome compositions of abundant genera were remarkably similar in full body and gut samples with only some genera having major quantitative differences. The most notable of these are the genera Lactococcus (gut: 54.71\%, SD: 47.32\%; full body: 1.56\%, SD: $2.58 \%$ ) and Providencia (gut: $39.35 \%$, SD: $48.34 \%$; full body: $77.51 \%$, SD: $41.41 \%)$ in the Argentinian population and Lysinibacillus (gut: $1.77 \%$, SD: $1.08 \%$; full body: $12.46 \%$, SD: $7.7 \%$ ) in the
Australian population. Beside this, there are only differences in low abundance genera (details are provided in Supplementary Table S11). Conversely, we observed considerable variation across populations. Argentina samples were dominated by Providencia (58.43\%) and Lactobacillus (28.13\%) complemented with a number of genera in low abundance. Australian samples are dominated by Bacillus (71.91\%) followed by Staphylococcus (11.94\%) and Lysinibacillus (7.11\%). Samples from Greece were dominated by Acinetobacter (98.63\%). Italian samples had the most even spread, dominated by Morganella (46.59\%), and Klebsiella (18.58\%) but having multiple genera with a significant presence [Providencia (5.27\%), Enterobacter (4.36\%), Lactobacillus (3.30\%), etc.]. Only a few genera, Providencia, Acinetobacter, Morganella, and Klebsiella, were presented across all populations. Details about the taxa relative abundance and variability are provided in Supplementary Table S11.

\section{DISCUSSION}

This study explored the effects of commonly used sample processing protocols on the gut microbiome of C. capitata recovered by amplicon sequencing of $16 \mathrm{~S} r R N A$ genes across life stages and samples from different sources. Our results showed that the gut microbiome of the target C. capitata colonies 

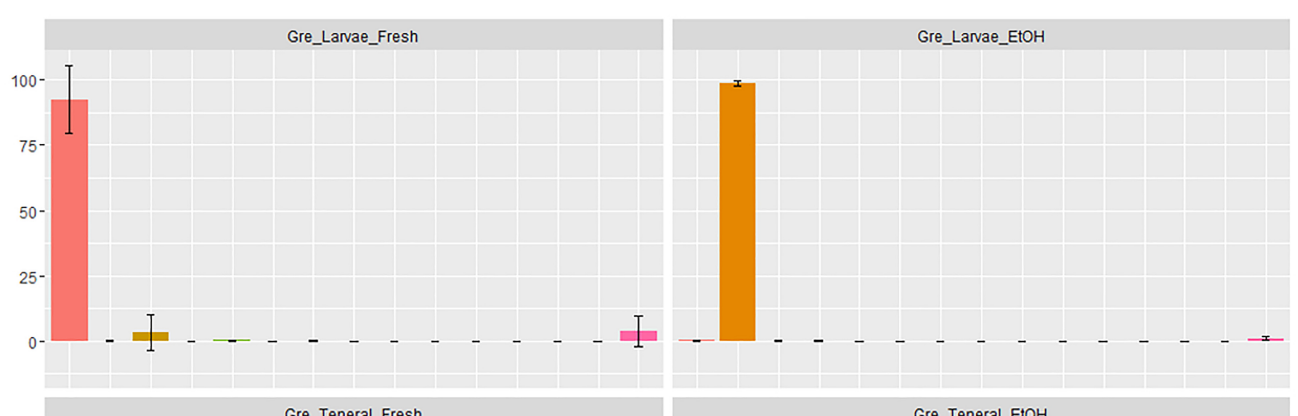

Genus
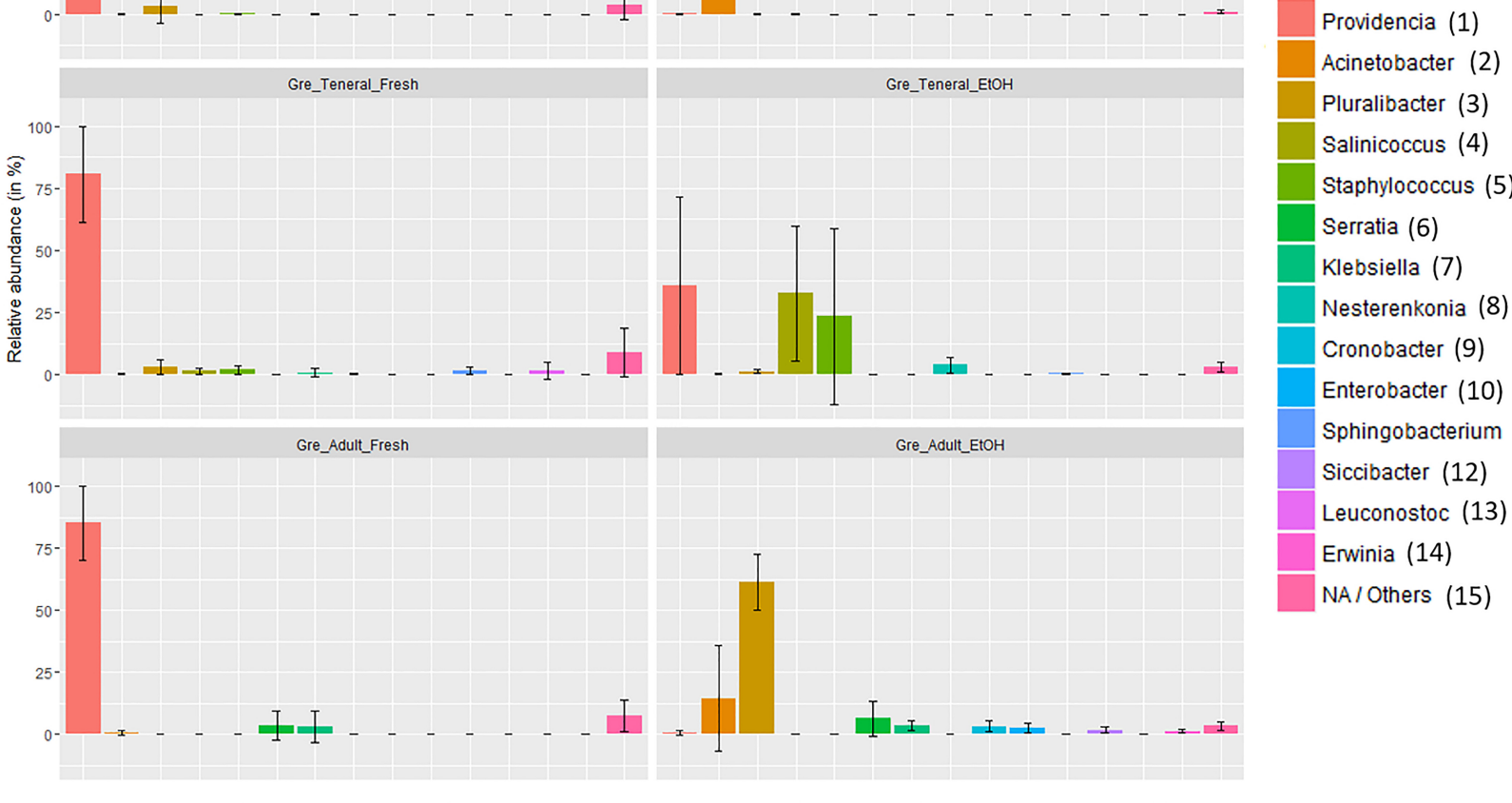

Sphingobacterium (11)

Siccibacter (12)

Leuconostoc (13)

Erwinia (14)

NA/ Others (15)

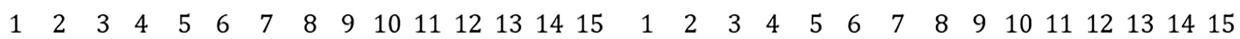

FIGURE 3 | Relative abundance of gut symbionts (genus level classification) in fresh and ethanol preserved specimens of larvae, teneral, and adults of $C$. capitata from the colony strain of Greek origin.

mainly consisted of members of the Proteobacteria ( $>91 \%$ of reads) and Firmicutes, and to a lesser extent, Bacteroidetes and Actinobacteria. The phylum of Proteobacteria was mainly composed of members of the Enterobacteriaceae family. This composition is compatible with other studies done in C. capitata and other tephritid fruit flies (Behar et al., 2008b; Prabhakar et al., 2013; Andongma et al., 2015; Yong et al., 2016). Comparing microbiome composition at genus level, we find that most samples studied in this experiment are dominated by only one or two genera. Although there is considerable variation across samples, one of the most dominant bacterial genera was Providencia (Supplementary Table S2). This genus has been commonly detected as a part of the gut microbiome of $C$. capitata and other fruit fly species (Allwood and Drew, 1996; Behar et al., 2008a; Ami et al., 2010; Wang et al., 2011; Augustinos et al., 2015; IAEA, 2016). In contrast to many other members from the Enterobacteriaceae family, this genus consists mainly of opportunistic pathogenic species (Boemare et al., 1996; Juneja and Lazzaro, 2009; Galac and Lazzaro, 2011), so it would be interesting to investigate the potential role of Providencia in medfly and find out if it acts as a beneficial or parasitic partner. Other dominant genera included Bacillus, Acinetobacter, Staphylococcus, and Morganella. All of these genera have been recorded in multiple earlier studies of the fruit fly gut microbiome (Kuzina et al., 2001; Alma, 2008; Thaochan et al., 2010; Wang et al., 2011; Yuval et al., 2013; Hadapad et al., 2015; IAEA, 2016; Liu et al., 2016; Yong et al., 2016).

From our comparison of different sample treatment and preservation protocols, we can draw multiple conclusions regarding the microbiome composition. First of all, we found that dissection of the gut seems to have little impact on the microbiome profiling. Across all methods used to compare gut dissection against the use of full bodies (e.g., diversity indexes, PERMANOVA, PERMDISP, PCOA, and taxonomic composition), we only found a significant differences between gut and full body microbiome profiles in fresh samples from Experiment 1 (see Supplementary Table S8a). However, analysis of the PCOA (Figure 2) and visual inspection of taxon composition (see Supplementary Table S10) suggested that this difference was related to a lower relative abundance of the most dominant genus, Providencia, in full body samples. While there were only minor differences in relative abundance in many 


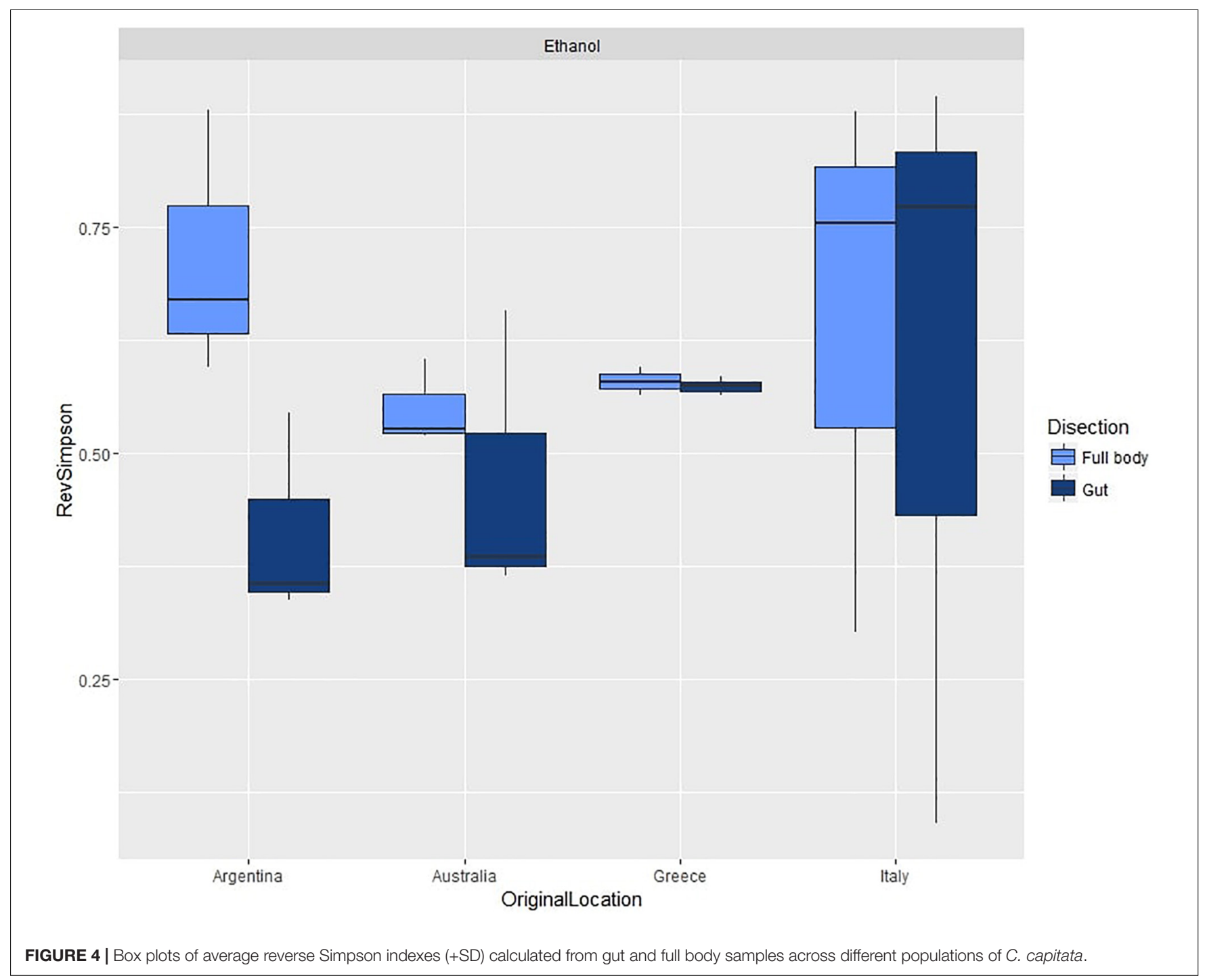

other genera. This results in similar patterns of composition to be found between gut dissection and use of full bodies. This relatively little difference observed between gut and full body microbiome profiles suggests that the gut bacterial community is predominant or outnumber bacteria occurring in the rest of the body and/or that bacterial communities end up mixing during dissection.

In contrast to the limited effect of dissection, preserving fruit flies in $70 \% \mathrm{EtOH}$ strongly affected the microbiota composition as revealed through $16 \mathrm{~S} r R N A$ gene amplicon sequencing. This is consistent with recent studies of the effects of $\mathrm{EtOH}$ preservation of different fecal samples (Hale et al., 2015; Sinha et al., 2016; Song et al., 2016). To our knowledge this is the first time the effects of $70 \% \mathrm{EtOH}$ preservation have been shown in insect tissue. The most prominent effect found in this experiment was an increase in the evenness of EtOH-preserved samples, as indicated by the Simpson index, through the decrease in abundance of Providencia, i.e., the most dominant genus observed in fresh specimens. This was consistently observed in all different life stages and is reflected in the comparison of the reverse Simpson index (see Table 1A and Figure 1). Seeing we encountered Providencia in much lower relative abundance in all EtOHpreserved samples, we detected other genera with higher relative abundances. Staphylococcus, Salinicoccus, Acinetobacter, Serratia, and Klebsiella. All had a low abundance in fresh samples but dominate EtOH-preserved samples. Beside these major changes, we also found minor changes for many genera. For some, this was a small decrease in relative abundance for $\mathrm{EtOH}$-preserved samples, while for others there was a small increase. For a number of genera, with a very low abundance, these small changes made the difference between being detected or not. It is likely that genera that have a decreased relative abundance in EtOH-preserved samples, such as Providencia, are negatively affected by EtOH preservation more than the other genera, while this decrease makes it easier for other genera to be detected. Hale et al. (2015) reported that in fecal samples preservation methods could exhibit this kind of bias toward or against certain microbiological groups. EtOH preservation does not only affect 


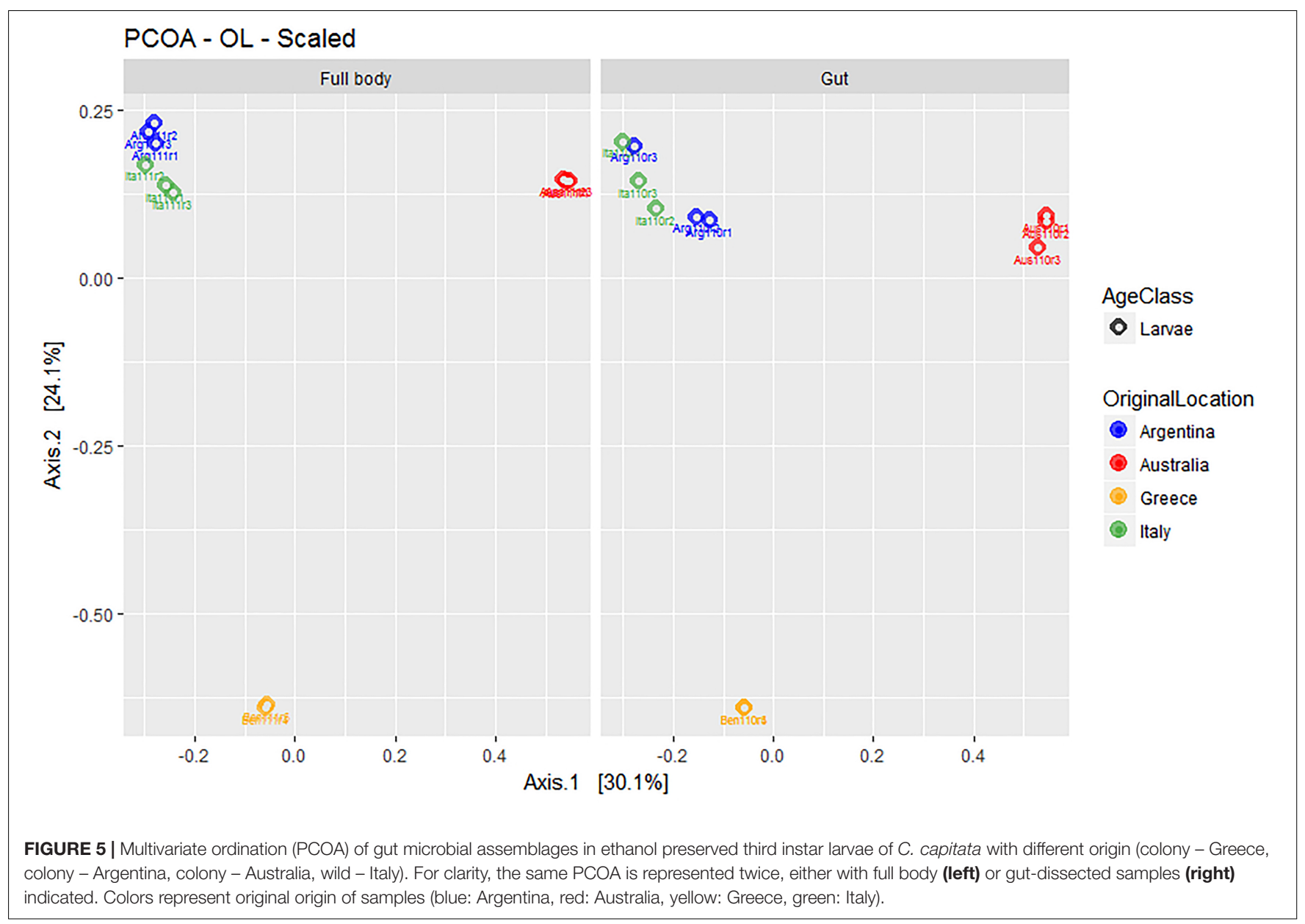

microbiome composition but also affects the variation between replicate samples.

In general, comparison of the different life stages showed that the gut microbiota of larvae is not only different in composition, but also have a lower diversity and variation when compared to adults and tenerals. This is not unexpected when taking in account the different feeding, lower mobility, and interaction with the environment of larvae in comparison with adults. However in larvae, we found that the multivariate dispersion in EtOH-preserved samples was much lower than in fresh samples. This is in contrast to what was found in tenerals and adults where fresh samples showed a lower dispersion. It is likely that this is linked to the complexity of the gut microbiome. In larvae, we found that after EtOH preservation samples are again completely dominated by one genus, Acinetobacter, while in tenerals and adults there is a more even spread with multiple genera being dominant. Additionally, in all EtOH-preserved samples differences in the relative abundances of a number of taxa could also be observed. The non-consistent patterns observed suggest that the use of $70 \% \mathrm{EtOH}$ as preservative might produce unpredictable effects on the microbiome profiles of samples, including not consistent shifts in the relative proportion of the less abundant bacterial taxa. However, further experimental validation is necessary to verify this hypothesis.
In all fresh samples we found comparable microbiome compositions even across life stages, which were all consistently dominated by members of the genus Providencia. As expected, many differences can be found when looking at the less abundant bacterial taxa and this seems the reason of the difference in microbial profiles between fresh tenerals and adults (see Supplementary Table S8a). Conversely, in EtOH-preserved samples we detected major differences across the different life stages. We hypothesize that the reduced abundance Providencia after EtOH preservation allows less abundant taxa to dominate or become detectable in the gut microbiome profiles from EtOHpreserved samples. This, and the added unpredictability, might magnify the differences between life stages, and showing no consistent differences in gut microbiome composition. Therefore, our tests on EtOH-preserved specimens seem to confirm earlier studies that shifts in the gut microbiome profiles occur across fruit fly development stages (Aharon et al., 2013; Andongma et al., 2015). These results should be taken cautiously as compositional changes observed in fresh specimens seem to be far less impressive.

The second experiment allowed us to verify the consistency of patterns observed for the first experiments on an heterogeneous group of fruit fly samples from different sources. In this experiment we observed high variability in both diversity and 


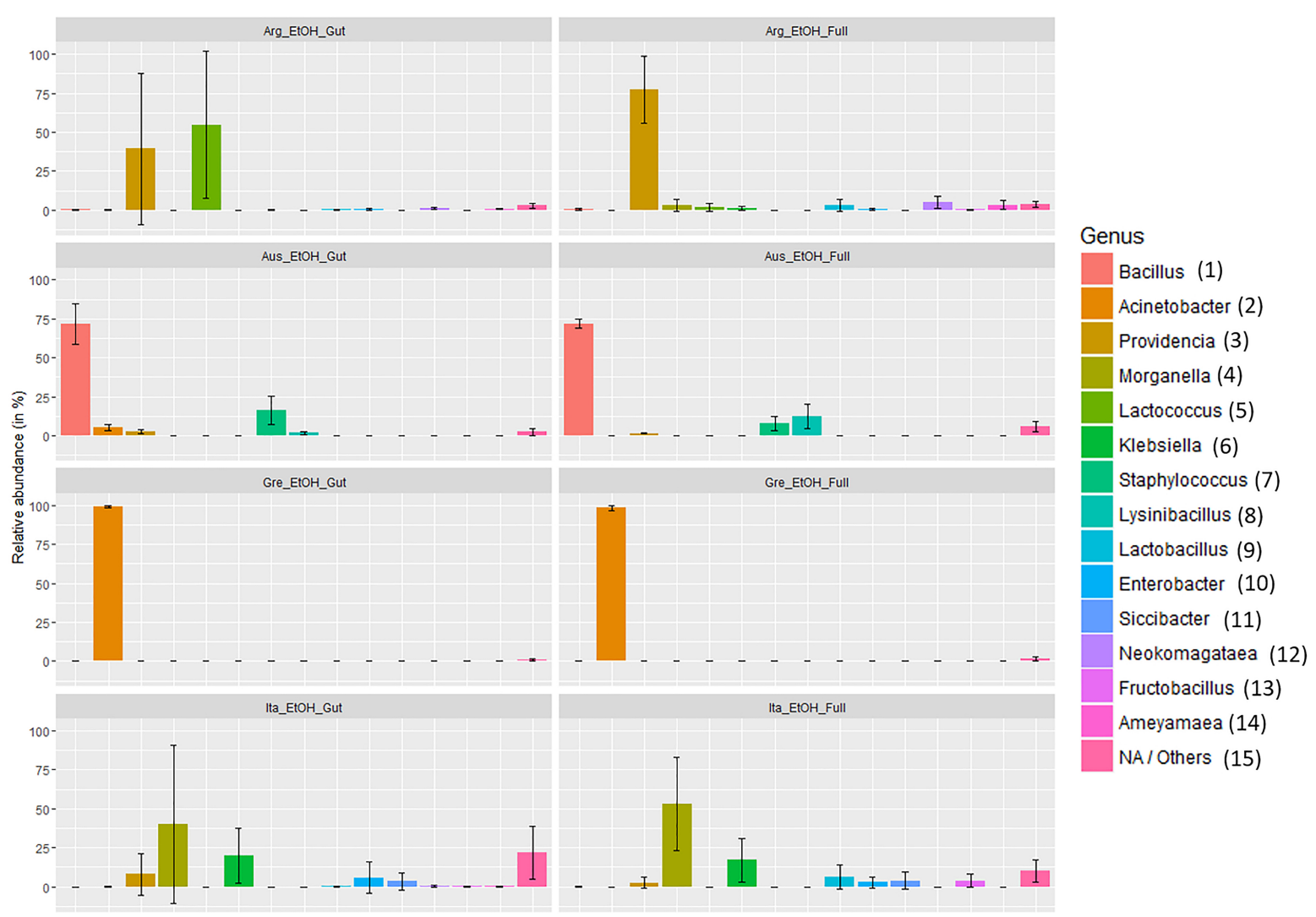

$\begin{array}{llllllllllllllllllllllllllllll}1 & 2 & 3 & 4 & 5 & 6 & 7 & 8 & 9 & 10 & 11 & 12 & 13 & 14 & 15 & 1 & 2 & 3 & 4 & 5 & 6 & 7 & 8 & 9 & 10 & 11 & 12 & 13 & 14 & 15\end{array}$

FIGURE 6 | Relative abundance of gut symbionts (genus level classification) in ethanol preserved third instar larvae of $C$. capitata from different samples (colony Greece, colony - Argentina, colony - Australia, wild - Italy).

composition of microbiome profiles (Table 1B). Even the Greek, Australian, and Argentinian laboratory populations, which were reared with an identical diet and similar environmental conditions, still had very distinct gut microbiome compositions. It is however difficult to speculate about the combination of processes responsible for the observed patterns. The geographical origin of samples seems to have a very strong effect on the gut microbiome composition; however, there also seemed that there is a correspondence between the colony age and the diversity within/variation between samples. The larvae from the (long established) Greek colony populations showed the lowest variation, followed by the (intermediately established) Australian colony population. The variation in the Italian (wild) population and (recently established) Argentinian colony population was much higher (see Supplementary Table S9b). This result seemed to be in line with results of previous studies comparing diversity and composition of the gut microbiome from colony and wild fruit fly populations (Tsiropoulos, 1983; Konstantopoulou et al., 1999; Ben-yosef et al., 2015; Morrow et al., 2015; Deutscher et al., 2018; Malacrinò et al., 2018). To adequately disentangle the effects of colony age and colony origin, targeted experiments with adequately replicated samples of similar ages and/or origins are needed.

\section{CONCLUSION}

In conclusion, we found that differences in gut microbial profiles obtained from gut dissected and non-dissected samples were only minor with patterns that were stable across all life stage and samples from different sources. In contrast, preservation of samples in $70 \% \mathrm{EtOH}$ had a major effect on the resulting gut microbiome profiles and was associated to higher inter-replicate variability and not consistent changes across life stages. These results shed new light on how samples preparation protocols can affect the results of HTS experiments and will help us interpreting and cross-compare the results of future and past studies. This study suggests that standardizing wet-lab procedures will increase the consistency, reliability, and repeatability of microbiomic research. A recommendation could be made against the use of $70 \% \mathrm{EtOH}$, a widely used preservatives in entomology, as the proportion of water is still probably too high to guarantee efficient gut microbiome fixation and preservation. When possible, fresh material or of more efficient preservative approaches (tentatively including deep freezing and absolute EtOH) should be preferred. Further experiments comparing different sample preparation protocols, different preservation techniques, on different model organisms, might give more insight in the use alternative sample 
preparation and preservation protocols. These studies will help us to further identify the effects of variations in the sample preparation and help pave the way a more comprehensive understanding of the insect gut microbiome.

\section{DATA AVAILABILITY STATEMENT}

The datasets generated for this study can be found in the NCBI Sequence Read Archive (https://www.ncbi.nlm.nih. gov/sra) and can be accessed with the accession numbers: SRR8732251-SRR8732302.

\section{AUTHOR CONTRIBUTIONS}

$\mathrm{MD}, \mathrm{AW}, \mathrm{MV}$, and PV designed the research and secured the funding. $\mathrm{MD}, \mathrm{AA}, \mathrm{KB}$, and $\mathrm{MV}$ designed and performed the experiments. MD and MV analyzed the data with input from all other authors. MD wrote the manuscript. All authors proofread, edited, and approved the manuscript.

\section{FUNDING}

This research was supported by the Belgian Federal Science Policy administration (BelSPO) BRAIN-be program (grant BR/154/PI/SYMDIV).

\section{ACKNOWLEDGMENTS}

We would like to thank Nathalie Smitz (RMCA) and Liesbeth Lebbe (LM-UGent) for guidance for the lab work and Alessandro de Sciscio and Thomas van den Wijngaert for the assistance with the practical lab procedures.

\section{SUPPLEMENTARY MATERIAL}

The Supplementary Material for this article can be found online at: https://www.frontiersin.org/articles/10.3389/fmicb. 2019.02833/full\#supplementary-material

\section{REFERENCES}

Abdi, H., and Williams, L. (2010). “Tukey's Honestly Signiflcant Difierence (HSD) Test," in Encyclopedia of Research Design, ed. N. J. Salkind (Thousand Oaks, CA: Sage Plubications), 1-7. Available at: https://www.researchgate.net/publication/ 237426041_Tukey's_Honestly_Signiflcant_Difierence_HSD_Test

Aharon, Y., Pasternak, Z., Ben-Yosef, M., Behar, A., Lauzon, C. R., Yuval, B., et al. (2013). Phylogenetic, metabolic, and taxonomic diversities shape mediterranean fruit fly microbiotas during ontogeny. Appl. Environ. Microbiol. 79, 303-313. doi: 10.1128/AEM.02761-12

Allwood, A. J., and Drew, R. A. I. (1996). Management of Fruit Flies in the Pacific. Canberra: Australian Centre for International Agricultural Research.

Alma, A. (2008). Insect Symbiosis, Vol. 3, eds. K. Bourtzis and T. A. Miller (New York: Taylor \& Francis Group).
TABLE S1 | Results of DNA barcoding identifications implemented on wild samples; identified species: Top matched species, Top\%: highest similarity with the identified species, Low\%: lowest similarity with the identified species.

TABLE S2 | Overview of the experimental setup: Experiment 1: three life stages (larvae, tenerals, and adults), two forms of preservation (fresh and preserved in $70 \%$ ethanol), and two forms of samples preparation (dissected and full body) across one population of $C$. capitata. Experiment 2: one life stage (larvae), two forms of preservation (fresh and preserved in $70 \%$ ethanol), and two forms of samples preparation (dissected and full body) across three different colony strains and one wild population.

TABLE S3 | List of the considered C. capitata samples for this study.

TABLE S4 | Detailed analytical pipeline implemented for data filtering and taxon assignment.

TABLE S5 | Overview of sequencing data and taxon assignment after data filtering.

TABLE S6 | Relative composition of groups in all samples of $C$. capitata included in this study. Inner circle: phylum composition; outer circle: most abundant genera.

TABLE S7 | ANOVA and a posteriori comparisons (Tukey's HSD test) testing for differences in patterns of alpha diversity (as estimated by the Reverse Simpson index calculated from OTU data) across (A) dissection procedures, sample preservation methods, and life stages of $C$. capitata and (B) dissection procedures on different populations of $C$. capitata. df, degrees of freedom; MS, mean square estimates; F, pseudo-F; P, $p$-value; n.s., not significant; and ***, at $P<0.001$. Tukey's HSD test: diff, difference in test scores; Iwr-upr, lower and upper limits of $95 \%$ confidence interval; $p$-adj, false discovery rate-adjusted $p$-value.

TABLE S8 | PERMANOVA and a posteriori comparisons (t-tests) verifying differences in OTUs abundance and composition across (A) dissection procedures, sample preservation methods, and life stages of $C$. capitata and (B) dissection procedures on different populations of C. capitata. df, degrees of freedom; MS, mean square estimates; F, pseudo- $F ; P, p$-value; n.s., not significant; and ${ }^{*}$, significant at $P<0.05$, ${ }^{* *}$, at $P<0.01$, ***, at $P<0.001$.

TABLE S9 | PERMDISP and a posteriori comparisons ( $t$-tests) verifying differences in variation within samples across (A) sample preservation methods and life stages of $C$. capitata and (B) dissection procedures on different populations of C. capitata. df, degrees of freedom; MS, mean square estimates; F, pseudo- $F$; P, $p$-value; n.s., not significant; and *, significant at $P<0.05$, **, at $P<0.01$, ***, at $P<0.001$.

TABLE S10 | Overview of average relative abundance (+SD) of the 15 most abundant genera in fresh and ethanol preserved specimens of reared larvae, teneral, and adults from the Greek laboratory colony of $C$. capitata.

TABLE S11 | Overview of average relative abundance (+SD) of the 15 most abundant genera in fresh and ethanol preserved third instar larvae of $C$. capitata with different origin.

Ami, E. B., Yuval, B., and Jurkevitch, E. (2010). Manipulation of the microbiota of mass-reared mediterranean fruit flies Ceratitis capitata (Diptera: Tephritidae) improves sterile male sexual performance. ISME J. 4, 28-37. doi: 10.1038/ismej. 2009.82

Anderson, M. J. (2006). Distance-based tests for homogeneity of multivariate dispersions. Biometrics 62, 245-253. doi: 10.1111/j.1541-0420.2005.00440.x

Anderson, M. J. (2017). "Permutational multivariate analysis of variance (PERMANOVA)," in Wiley StatsRef: Statistics Reference Online, eds N. Balakrishnan, T. Colton, B. Everitt, W. Piegorsch, F. Ruggeri, and J. L. Teugels, (Hoboken, NJ: Wiley), 1-15. doi: 10.1002/9781118445112.stat07841

Andongma, A. A., Wan, L., Dong, Y., Li, P., Desneux, N., White, J. A., et al. (2015). Pyrosequencing reveals a shift in symbiotic bacteria populations across life stages of Bactrocera dorsalis. Sci. Rep. 5:9470. doi: 10.1038/srep0 9470 
Andrews, S. (2014). FastQC A Quality Control Tool for High Throughput Sequence Data. Available at: http://www.bioinformatics.babraham.ac.uk/projects/fastqc/ (accessed January 1, 2014).

Augustinos, A. A., Kyritsis, G. A., Papadopoulos, N. T., Abd-Alla, A. M. M., Cáceres, C., and Bourtzis, K. (2015). Exploitation of the medfly gut microbiota for the enhancement of sterile insect technique: use of Enterobacter Sp. in larval diet-based probiotic applications. PLoS One 10:e0136459. doi: 10.1371/journal. pone. 0136459

Behar, A., Jurkevitch, E., and Yuval, B. (2008a). Bringing back the fruit into fruit flybacteria interactions. Mol. Ecol. 17, 1375-1386. doi: 10.1111/j.1365-294X.2008. 03674.x

Behar, A., Yuval, B., and Jurkevitch, E. (2008b). Gut bacterial communities in the mediterranean fruit fly (Ceratitis capitata) and their impact on host longevity. J. Insect Physiol. 54, 1377-1383. doi: 10.1016/j.jinsphys.2008.07.011

Benjamini, Y., and Hochberg, Y. (1995). Controlling the false discovery rate: a practical and powerful approach to multiple testing. J. R. Stat. Soc. B 57, 289-300. doi: 10.1111/j.2517-6161.1995.tb02031.x

Ben-yosef, M., Pasternak, Z., Jurkevitch, E., Yuval, B., and Ben-yosef, M. (2015). Symbiotic bacteria enable olive fly larvae to overcome host defences. $R$. Soc. Open Sci. 2:150170. doi: 10.1098/rsos.150170

Boemare, N., Laumind, C., and Maulon, H. (1996). The entomopathogenic nematode-bacterium complex: biology, life cycle and vertebrate safety. Biocontrol Sci. Technol. 6, 333-346. doi: 10.1080/09583159631316

Bray, J. R., and Curtis, J. T. (1957). An ordination of the upland forest communities of southern wisconsin. Ecol. Monogr. 27, 325-349. doi: 10.2307/1942268

Callahan, B. J., McMurdie, P. J., Rosen, M. J., Han, A. W., Johnson, A. J. A., and Holmes, S. P. (2016). DADA2: high-resolution sample inference from illumina amplicon data. Nat. Methods 13, 581-583. doi: 10.1038/nmeth.3869

Ceja-Navarro, J. A., Vega, F. E., Karaoz, U., Hao, Z., Jenkins, S., Lim, H. C., et al. (2015). Gut microbiota mediate caffeine detoxification in the primary insect pest of coffee. Nat. Commun. 6, 1-9. doi: 10.1038/ncomms8618

Cheng, D., Guo, Z., Riegler, M., Xi, Z., Liang, G., and Xu, Y. (2017). Gut symbiont enhances insecticide resistance in a significant pest, the oriental fruit fly Bactrocera dorsalis (Hendel). Microbiome 5:13. doi: 10.1186/s40168-0170236-z

Clarke, A. R. (2016). Why so many polyphagous fruit flies (Diptera: Tephritidae)? A further contribution to the 'generalism' debate. Biol. J. Linn. Soc. 120, 245-257. doi: 10.1111/bij.12880

Cole, J. R., Wang, Q., Fish, J. A., Chai, B., McGarrell, D. M., Sun, Y., et al. (2014). Ribosomal database project: data and tools for high throughput RRNA analysis. Nucleic Acids Res. 42, 633-642. doi: 10.1093/nar/gkt1244

Colman, D. R., Toolson, E. C., and Takacs-Vesbach, C. D. (2012). Do diet and taxonomy influence insect gut bacterial communities? Mol. Ecol. 21, 5124-5137. doi: 10.1111/j.1365-294X.2012.05752.x

de Cárcer, D. A., Denman, S. E., McSweeney, C., and Morrison, M. (2011). Evaluation of subsampling-based normalization strategies for tagged highthroughput sequencing data sets from gut microbiomes. Appl. Environ. Microbiol. 77, 8795-8798. doi: 10.1128/AEM.05491-11

De Meyer, M., Robertson, M. P., Peterson, A. T., and Mansell, M. W. (2008). Ecological niches and potential geographical distributions of mediterranean fruit fly (Ceratitis capitata) and natal fruit fly (Ceratitis rosa). J. Biogeogr. 35, 270-281. doi: 10.1111/j.1365-2699.2007.01769.x

DeSantis, T. Z., Hugenholtz, P., Larsen, N., Rojas, M., Brodie, E. L., Keller, K., et al. (2006). Greengenes, a chimera-checked 16S RRNA gene database and workbench compatible with ARB. Appl. Environ. Microbiol. 72, 5069-5072. doi: 10.1128/AEM.03006-05

Deutscher, A. T., Burke, C. M., Darling, A. E., Riegler, M., Reynolds, O. L., and Chapman, T. A. (2018). Next generation sequencing revealed Asaia as a common midgut bacterium of wild and domesticated queensland fruit fly larvae. Microbiome 6:85. doi: 10.1186/s40168-018$0463-y$

Dillon, R. J., and Dillon, V. M. (2004). The gut bacteria of insects: nonpathogenic interactions. Annu. Rev. Entomol. 49, 71-92. doi: 10.1146/annurev.immunol.20. 081501.130710

Dominiak, B. C., and Daniels, D. (2012). Review of the past and present distribution of Mediterranean fruit fly (Ceratitis capitata Wiedemann) and Queensland fruit fly (Bactrocera tryoni Froggatt) in Australia. Aust. J. Entomol. 51, 104-115. doi: $10.1111 / j .1440-6055.2011 .00842 . x$
Engel, P., and Moran, N. A. (2013). The gut microbiota of insects - diversity in structure and function. FEMS Microbiol. Rev. 37, 699-735. doi: 10.1111/15746976.12025

Galac, M. R., and Lazzaro, B. P. (2011). Comparative pathology of bacteria in the genus providencia to a natural host, Drosophila melanogaster. Microbes Infect. 13, 673-683. doi: 10.1016/j.micinf.2011.02.005

Garofalo, C., Osimani, A., Milanović, V., Taccari, M., Cardinali, F., Aquilanti, L., et al. (2017). The microbiota of marketed processed edible insects as revealed by high-throughput sequencing. Food Microbiol. 62, 15-22. doi: 10.1016/j.fm. 2016.09.012

Gavriel, S., Jurkevitch, E., Gazit, Y., and Yuval, B. (2011). Bacterially enriched diet improves sexual performance of sterile male Mediterranean fruit flies. J. Appl. Entomol. 135, 564-573. doi: 10.1111/j.1439-0418.2010.01605.x

Hadapad, A. B., Prabhakar, C. S., Chandekar, S. C., Tripathi, J., and Hire, R. S. (2015). Diversity of bacterial communities in the midgut of Bactrocera cucurbitae (Diptera: Tephritidae) populations and their potential use as attractants. Pest Manag. Sci. 72, 1222-1230. doi: 10.1002/ps.4102

Hale, V. L., Tan, C. L., Knight, R., and Amato, K. R. (2015). Effect of preservation method on spider monkey (Ateles geoffroy) fecal microbiota over 8 weeks. J. Microbiol. Methods 113, 16-26. doi: 10.1016/j.mimet.2015.03.021

Hammer, T. J., Dickerson, J. C., and Fierer, N. (2015). Evidence-based recommendations on storing and handling specimens for analyses of insect microbiota. PeerJ 3:e1190. doi: 10.7717/peerj.1190

Holguin, G., Vazquez, P., and Bashan, Y. (2001). The role of sediment microorganisms in the productivity, conservation, and rehabilitation of mangrove ecosystems: an overview. Biol. Fertil. Soils 33, 265-278. doi: 10.1007/ s003740000319

Husseneder, C., and Grace, J. K. (2005). Genetically engineered termite gut bacteria (Enterobacter cloacae) deliver and spread foreign genes in termite colonies. Appl. Microbiol. Biotechnol. 68, 360-367. doi: 10.1007/s00253-005-1914-5

IAEA, (2016). Use of Symbiotic Bacteria to Reduce Mass-Rearing Cost and Increase Mating Success in Selected Fruit Pests in Support of SIT Application. Vienna: IAEA.

Juneja, P., and Lazzaro, B. P. (2009). Providencia sneebia Sp. nov. and Providencia burhodogranariea Sp. Nov., isolated from wild Drosophila melanogaster. Int. J. Syst. Evol. Microbiol. 59, 1108-1111. doi: 10.1099/ijs.0.000117-0

Kinross, J. M., Darzi, A. W., and Nicholson, J. K. (2011). Gut microbiome-host interactions in health and disease. Genome Med. 3:14. doi: 10.1186/gm228

Konstantopoulou, M. A., Economopoulos, A. P., and Raptopoulos, D. G. (1999). Artificial rearing antimicrobials as selecting factors of adhalleles in Bactrocera(Dacus) oleae(Diptera: Tephritidae). J. Econ. Entomol. 92, 563-568. doi: 10.1093/jee/92.3.563

Kuzina, L. V., Peloquin, J. J., Vacek, D. C., and Miller, T. A. (2001). Isolation and identification of bacteria associated with adult laboratory mexican fruit flies, Anastrepha ludens (Diptera: Tephritidae). Curr. Microbiol. 42, 290-294. doi: $10.1007 / \mathrm{s} 002840110219$

Lande, R. (2016). Statistics and partitioning of species diversity, and similarity among multiple communities. Nordic Soc. Oikos 76, 5-13.

Liu, L. J., Martinez-Sañudo, I., Mazzon, L., Prabhakar, C. S., Girolami, V., Deng, Y. L., et al. (2016). Bacterial communities associated with invasive populations of Bactrocera dorsalis (Diptera: Tephritidae) in China. Bull. Entomol. Res. 106, 718-728. doi: 10.1017/S0007485316000390

Malacrinò, A., Campolo, O., Medina, R. F., and Palmeri, V. (2018). Instar- and host-associated differentiation of bacterial communities in the Mediterranean fruit fly Ceratitis capitata. PLoS One 13:e0194131. doi: 10.1371/journal.pone. 0194131

McMurdie, P. J., and Holmes, S. (2013). Phyloseq: an R Package for reproducible interactive analysis and graphics of microbiome census data. PLoS One 8:e61217. doi: 10.1371/journal.pone.0061217

Metzker, M. L. (2010). Sequencing technologies the next generation. Nat. Rev. Genet. 11, 31-46. doi: 10.1038/nrg2626

Morales-Jiménez, J., Zúñiga, G., Ramírez-Saad, H. C., and Hernández-Rodríguez, C. (2012). Gut-associated bacteria throughout the life cycle of the bark beetle Dendroctonus Rhizophagus thomas and bright (Curculionidae: Scolytinae) and their cellulolytic activities. Microb. Ecol. 64, 268-278. doi: 10.1007/s00248-0119999-90

Morey, M., Fernández-Marmiesse, A., Castiñeiras, D., Fraga, J. M., Couce, M. L., and Cocho, J. A. (2013). A glimpse into past, present, and future 
DNA sequencing. Mol. Genet. Metab. 110, 3-24. doi: 10.1016/j.ymgme.2013. 04.024

Morrow, J. L., Frommer, M., Shearman, D. C. A., and Riegler, M. (2015). The microbiome of field-caught and laboratory-adapted Australian Tephritid fruit fly species with different host plant use and specialisation. Microb. Ecol. 70, 498-508. doi: 10.1007/s00248-015-0571

NIH HMP Working Group, Peterson, J., Garges, S., Giovanni, M., McInnes, P., Wang, L., et al. (2009). The NIH human microbiome project. Genome Res. 19, 2317-2323. doi: 10.1101/gr.096651.109

Oksanen, J., Blanchet, F. G., Friendly, M., Kindt, R., Legendre, P., Mcglinn, D., et al. (2017). R Package 'Vegan ' [*Software]. Available at: https://cran.r-project.org/ web/packages/vegan/vegan.pdf.

Prabhakar, C. S., Sood, P., Kanwar, S. S., Sharma, P. N., Kumar, A., and Mehta, P. K. (2013). Isolation and characterization of gut bacteria of fruit fly, bactrocera tau (Walker). Phytoparasitica 41, 193-201. doi: 10.1007/s12600-012-0278-5

Pruesse, E., Peplies, J., and Glöckner, F. O. (2012). SINA: accurate high-throughput multiple sequence alignment of ribosomal RNA genes. Bioinformatics 28, 1823 1829. doi: 10.1093/bioinformatics/bts252 doi: 10.1093/bioinformatics/bts 252

Raymann, K., and Moran, N. A. (2018). The role of the gut microbiome in health and disease of adult honey bee workers. Curr. Opin. Insect Sci. 26, 97-104. doi: 10.1016/j.cois.2018.02.012

Rosenberg, E., Koren, O., Reshef, L., Efrony, R., and Zilber-Rosenberg, I. (2007). The role of microorganisms in coral health, disease and evolution. Nat. Rev. Microbiol. 5, 355-362. doi: 10.1038/nrmicro1635

Sinha, R., Chen, J., Amir, A., Vogtmann, E., Shi, J., Inman, K. S., et al. (2016). Collecting fecal samples for microbiome analyses in epidemiology studies. Cancer Epidemiol. Biomarkers Prev. 25, 407-416. doi: 10.1158/1055-9965.EPI15-0951

Song, S. J., Amir, A., Metcalf, J. L., Amato, K. R., Xu, Z. Z., Humphrey, G., et al. (2016). Preservation methods differ in fecal microbiome stability, affecting suitability for field studies. mSystems 1:e00021-16.

Takahashi, S., Tomita, J., Nishioka, K., Hisada, T., and Nishijima, M. (2014). Development of a prokaryotic universal primer for simultaneous analysis of bacteria and archaea using next-generation sequencing. PLoS One 9:e105592. doi: 10.1371/journal.pone.0105592

Thaochan, N., Drew, R. A. I., Hughes, J. M., Vijaysegaran, S., and Chinajariyawong, A. (2010). Alimentary tract bacteria isolated and identified with API-20E and molecular cloning techniques from Australian tropical fruit flies, Bactrocera cacuminata and B. tryoni. J. Insect Sci. 10:131. doi: 10.1673/031.010.13101

Tsiropoulos, G. J. (1983). Microflora associated with wild and labo- ratory reared adult olive fruit flies, Dacus oleae. Z. Angew. Entomol. 96, 337-340. doi: 10.1111/ j.1439-0418.1983.tb03680.x

Uchôa, M. A. (2014). Fruit flies (Diptera: Tephritoidea): biology, host plants, natural enemies, and the implications to their natural control. Integr. Pest Manag. Pest Control Curr. Future Tactics 2, 271-300. doi: 10.5772/ 31613

Virgilio, M., Jordaens, K., Breman, F. C., Backeljau, T., and de Meyer, M. (2012). Identifying insects with incomplete DNA barcode libraries, African Fruit Flies (Diptera: Tephritidae) as a test case. PLoS One 7:e31581. doi: 10.1371/journal. pone.0031581
Vlčková, K., Mrázek, J., Kopečn $\imath$, J., and Petrželková, K. J. (2012). Evaluation of different storage methods to characterize the fecal bacterial communities of captive Western Lowland Gorillas (Gorilla Gorilla Gorilla). J. Microbiol. Methods 91, 45-51. doi: 10.1016/j.mimet.2012.07.015

Wang, H., Jin, L., Peng, T., Zhang, H., Chen, Q., and Hua, Y. (2014). Identification of cultivable bacteria in the intestinal tract of Bactrocera dorsalis from three different populations and determination of their attractive potential. Pest. Manag. Sci. 70, 80-87. doi: 10.1002/ps.3528

Wang, H., Jin, L., and Zhang, H. (2011). Comparison of the diversity of the bacterial communities in the intestinal tract of adult Bactrocera dorsalis from three different populations. J. Appl. Microbiol. 110, 1390-1401. doi: 10.1111/j.13652672.2011.05001.x

Wang, Q., Garrity, G. M., Tiedje, J. M., and Cole, J. R. (2007). Naîve bayesian classifier for rapid assignment of RRNA sequences into the new bacterial taxonomy. Appl. Environ. Microbiol. 73, 5261-5267. doi: 10.1128/AEM.0006207

White, I. M., and Elson-Harris, M. M. (1992). Fruit Flies of Economic Significance: Their Identification and Bionomics. Wallingford: CAB International.

Wickham, H. (2009). Ggplot2: Elegant Graphics for Data Analysis. New York, NY: Springer.

Wong, C. N. A., Ng, P., and Douglas, A. E. (2011). Low-diversity bacterial community in the gut of the fruitfly Drosophila melanogaster. Environ. Microbiol. 13, 1889-1900. doi: 10.1111/j.1462-2920.2011.02511.x

Yong, H., Song, S., Chua, K., and Lim, P. (2016). Microbiota asociated with Bactrocera carambolae and $B$. dorsalis (Insecta: Tephritidae) revealed by nextgeneration sequencing of 16S RRNA Gene. Meta Gene 11, 189-196. doi: 10. 1016/j.mgene.2016.10.009

Yuval, B., Ben-Ami, E., Behar, A., Ben-Yosef, M., and Jurkevitch, E. (2013). The Mediterranean fruit fly and its bacteria - potential for improving sterile insect technique operations. J. Appl. Entomol. 137, 39-42. doi: 10.1111/j.1439-0418. 2010.01555.x

Zhang, J., Zhang, Y., Li, J., Liu, M., and Liu, Z. (2016). Midgut transcriptome of the cockroach periplaneta americana and its microbiota: digestion, detoxification and oxidative stress response. PLoS One 11:e0155254. doi: 10.1371/journal. pone.0155254

Zilber-Rosenberg, I., and Rosenberg, E. (2008). Role of microorganisms in the evolution of animals and plants: the hologenome theory of evolution. FEMS Microbiol. Rev. 32, 723-735. doi: 10.1111/j.1574-6976.2008.00123.x

Conflict of Interest: The authors declare that the research was conducted in the absence of any commercial or financial relationships that could be construed as a potential conflict of interest.

Copyright (c) 2019 De Cock, Virgilio, Vandamme, Augustinos, Bourtzis, Willems and De Meyer. This is an open-access article distributed under the terms of the Creative Commons Attribution License (CC BY). The use, distribution or reproduction in other forums is permitted, provided the original author(s) and the copyright owner(s) are credited and that the original publication in this journal is cited, in accordance with accepted academic practice. No use, distribution or reproduction is permitted which does not comply with these terms. 